\title{
Study of Performance Equivalent Simplification Analysis and Application of Stochastic Petri Nets
}

\author{
Da-bin Qi \\ University of Science and Technology Liaoning \\ Anshan China \\ qdbinner@163.com
}

\author{
Qiu-ju Li \\ Anshan Normal University \\ Anshan China \\ Liqiuju2013@163.com
}

\begin{abstract}
State spaces of Stochastic Petri Nets (SPN) are exponent explosion based on subordinate model's quantities, it is feasible to solve state space explosion. This article introduces basic theories of SPN performance equivalence simplification, integrates the real workflow of sanction management, constructs workflow model based on SPN comparatively, further more, simplifies the model by equivalence simplification theories, reduces model's complexity, solves the model performance quantity analysis effective.
\end{abstract}

Keywords-Stochastic Petri Nets; Performance analysis; Performance equivalence

\section{INTRODUCTION}

Stochastic Petri Nets(SPN) is Proposed and developped from 1981 to now, it has become the powerful tool of discrete events dynamic system in description and analysis aspect, it acquired prominent success in performance evaluation . for the complex systems model, application of SPN have some difficulties in complexity of models state space, it will have a exponential increase with system scale . Solving explosion problem of state space, equivalent commutation is a efficient method for SPN, by means, we can attain to reduce state space and simplified analysis .

The paper is based on problem, constructs workflow models , and integrates the real workflow of sanction management, constructs SPN models. Via equivalence simplification theories, the paper simplifies and analyzes model's performance.

\section{SPN PERFormance EQUivalence SimplicATION THEORIES}

Basic petri-nets models didn't include time parameters, so it had some difficulties in analyzing system performance. SPN contacts a random delay time which obeys exponential distribution , at the same time, the time is situated between each transition implementation and no implementation .it can enhace system performance analysis which the time contacts transition. SPN includes serial v parallel v choice and circulation structure. Equivalent simplification theories include:

Each basic structure as a sub network, using a time transition expresses equivalently basic structure time performance.

\section{A. Theory 1}

Serial structure performance equivalence simplification such as Figure 1, presuming the delay time of transition is " $\mathrm{n}$ " independent random variable, and complied with exponential distribution, which parameter is so as $\lambda_{1}, \lambda_{2}, \cdots, \lambda_{n}$, namely the average delay time of $\mathrm{n}$ serial transition is $\frac{1}{\lambda_{1}}, \frac{1}{\lambda_{2}}, \ldots, \frac{1}{\lambda_{n}}$, then its general average equivalence delay time is :

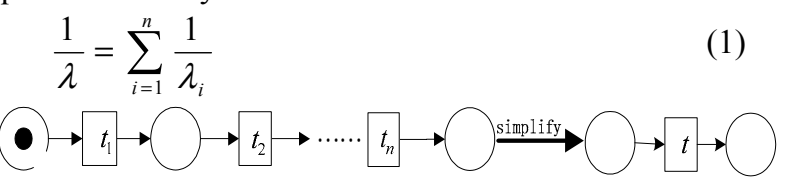

Figure 1. Serial structure performance equivalence simplification model

\section{B. $\quad$ Theory 2}

Parallel structure performance equivalence simplification such as Figure 2, presuming the delay time of transition is $\mathrm{n}$ independent sequence statistical variable $X_{1}, X_{2}, \cdots, X_{n}$, and complied with exponential distribution, which parameter is so as $\lambda_{1}, \lambda_{2}, \cdots, \lambda_{n}$, then its general average equivalence delay time is :

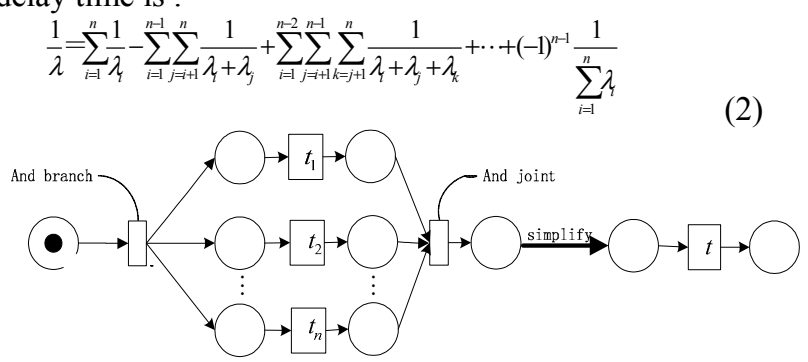

Figure 2. Parallel structure performance equivalence simplification model

\section{Theory 3}

Choice structure performance equivalence simplification such as Figure 3, presuming the delay time of transition is $n$ independent sequence statistical variable $\hat{t}_{1}, \hat{t}_{2}, \cdots, \hat{t}_{n}$, and complied with exponential distribution, which parameter is so as $\lambda_{1}, \lambda_{2}, \cdots, \lambda_{n}$, and the probability of executing 
transition $t_{i}: a_{i}, \sum_{i=1}^{n} a_{i}=1$, then its general average equivalence delay time :

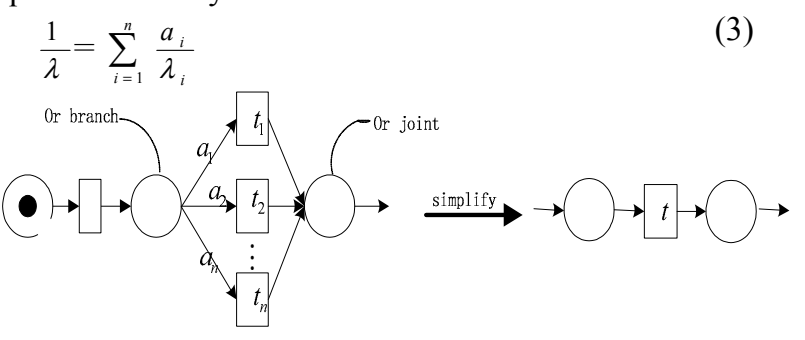

Figure 3. Choice structure performance equivalence simplification model

\section{Theory 4}

Circulation structure performance equivalence simplification such as Figure 4, presuming the delay time of two choice transition $\left(t_{1}, t_{2}\right)$ is two independent sequence statistical variable $\left(\hat{t}_{1}, \hat{t}_{2}\right)$, and complied with exponential distribution, which parameter is so as $\lambda_{1}, \lambda_{2}$, and presuming the executing transition $\left(t_{1}\right)$ is over, returning the probability of circulation executing transition $\left(t_{2}\right)$ is $a$, then its general average equivalence delay time:

$$
\frac{1}{\lambda}=\frac{\lambda_{2}+a \lambda_{1}}{(1-a) \lambda_{1} \lambda_{2}}
$$

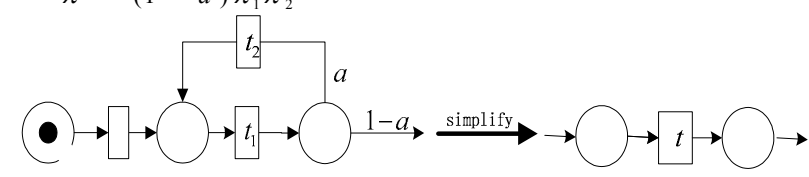

Figure 4. Circulation structure performance equivalence simplification model

\section{ANALYSIS Of APPLICATION PROBLEM}

\section{A. Sanction and management subsystem workflow analysis}

Sanction process of OA system commences on submitting, transacting, and pigeonholing in the end the process concludes 12 states: submit document、sanction、 transact 、 modify 、 verify 、 tidy 、 upload 、 check 、 sign 、 report 、 filing 、 pigeonhole. Via analyzing, submit document 、 sanction 、 transaction and check document execute serial structure, and modification and verification demand parallel structure, sign and upload report demand choice structure, sanction process may have two results: approve or refuse . system can send email to proposer , inform of repeating application after refusing. Refusing process may use circulation structure. The workflow model of sanction and management is such as Figure 5

\section{B. SPN performance equivalence simplification analysis}

The chart of utilizing workflow model to transform petri nets is such as Figure 5. Transform the role to transition, and transform submit document to start-up condition, transform pigeonhole to end-up condition, the conditions are expressed by place, in SPN, module of executing time is expressed by time transition, module of no executing time is expressed by moment transition (ignoring), and the method has no effect on final conclusion. Workflow model basing on SPN is such as Figure 6.

Based on executing state in upper system, presume time transition is $t_{0}, t_{1}, t_{2}, t_{3}, t_{4}, t_{5}, t_{6}$, the average delay time (unit is minute) :

$$
\frac{1}{\lambda_{0}}=30, \frac{1}{\lambda_{1}}=10, \frac{1}{\lambda_{2}}=120, \frac{1}{\lambda_{3}}=80, \frac{1}{\lambda_{4}}=60, \frac{1}{\lambda_{5}}=40, \frac{1}{\lambda_{6}}=20
$$

based on correlative theorem of exponential distribution function, presume the probability of no passing sanction : $a_{1}=0.8$, the probability of conjunct sign : $a_{2}=0.25$.Apply SPN performance equivalence simplification theories to simplify model, calculates from inner to outside, from complexity to simpleness, figures out performance parameter of the system. Simplification process is as follows :

1). $p_{8}, p_{9}, t_{5}, t_{6}$ and adjacent place construct choice structure. $t_{5}, t_{6}$ can simplify equivalently transition $t_{56}$ ( Figure 7), $\lambda_{56}$ expresses the reciprocal of new transition delay time(the same as follows), based on formula (3):

$\frac{1}{\lambda_{56}}=\frac{a_{2}}{\lambda_{5}}+\frac{1-a_{2}}{\lambda_{6}}=0.25 \times 40+0.75 \times 20=25$

2). $p_{2}, p_{3}, p_{4}, p_{5}, p_{6}, p_{7}, t_{2}, t_{3}, t_{4}$ and adjacent place construct parallel structure. $t_{2}, t_{3}, t_{4}$ can simplify equivalently transition $t_{234}$, based on formula

$$
\begin{aligned}
& \frac{1}{\lambda_{234}}=\frac{1}{\lambda_{2}}+\frac{1}{\lambda_{3}}+\frac{1}{\lambda_{4}}-\frac{1}{\lambda_{2}+\lambda_{3}}-\frac{1}{\lambda_{3}+\lambda_{4}}-\frac{1}{\lambda_{2}+\lambda_{4}} \\
& +\frac{1}{\lambda_{2}+\lambda_{3}+\lambda_{4}}=120+80+60-48-240 / 7-40 \\
& +80 / 3=164.381
\end{aligned}
$$

3). $p_{0}, p_{1}, t_{0}, t_{1}$ and adjacent place construct circulating structure. ${ }^{t_{0}, t_{1}}$ can simplify equivalently transition ${ }^{t_{01}}$, based on formula (4):

$$
\frac{1}{\lambda_{01}}=\frac{1}{1-a_{1}}\left(\frac{a_{1}}{\lambda_{0}}+\frac{1}{\lambda_{1}}\right)=5 \times(0.8 \times 30+10)=170
$$

4). Figure 7 is serial structure, $t_{01}, t_{234}, t_{56}$ can simplify equivalently transition $t_{0123456}$, based on formula (1), calculate general performance equivalence time :

$$
\frac{1}{\lambda_{0123456}}=\frac{1}{\lambda_{01}}+\frac{1}{\lambda_{234}}+\frac{1}{\lambda_{56}}
$$

$=170+164.381+25=359.381$

5). Based on general performance equivalence time, calculate other performance parameter, for instance, throughput the average transacting roles in unit time, etc. only a token, so throughput is reciprocal of equivalence time, namely 


$$
\lambda_{0123456}=0.00278(\text { transition } / \min u t e) \text { the average }
$$
transacting roles one day $24 \times 60 \times 0.00278=4.003$ (unit)

\section{CONCLUSION}

the paper applies equivalence simplification theories of SPN to simplify and analyze system performance. In the complicated OA system, concludes non-basic structure, transforms non-basic structure into basic structure, system design and function can attain the optimal state, the more application of the technology demands to study and explore.

\section{REFERENCES}

[1] K Salimifard,M Wright.Petri net-based modelling of workflow systems:An overview[J].European Journal of Operational Research,2001,134(3).

[2] Jiang Hao , Dong Yisheng, Luo Junzhou . Research on Petri Net Based Modeling and Analyzing Methods for Workflow Process [J]. Journal of Southeast University , 2000[2]: 68-70 (in Chinese)

[3] WFMC-TC-1011. Workflow Management Coalition Terminology\&Glossary[S]

[4] Jang G W.K J, Kim Y Y. Integrated topology and shape optimitation software for compliant MEMS mechanism design[J]. Advances in Engineering Software, 2008, 39 (1) : 1-12
[5] Li J H, Gao S M, Liu Y S. Feature-based process layer modeling for surface micromachined MEMS [J]. Journal of Micromechanics and Microengineering, 2005,15 (3) : 620-630

[6] Girault C, Valk R Petri nets for Systems Engineering: A Guide to Modeling, Verification, and Applications. Berlin: Springer-Verlag, 2003 483-565

[7] Lerbet J. About the synchronization of MEMs [J].Nonlinear Analysis : Real World Applications, 2009,10(1) : 266-276

[8] Kim G H, Park K C. A continuum-based modeling of MEMS devices for estimating their resonant frequencies[J]. Computer Methods in Applied Mechanics and Engineering, 2008, 198(2) : $234-242$

[9] YUAN Chongyi. The principle and application of Petri nets[M].Beijing : Publishing House of Electronics Industry, 2005.

[10] KIM, H W, KIM Y G. Dynamic process modeling for BPR : a computerized simulation approach $[\mathrm{J}]$. Information and Management, 1997,32(1) : 1-13

[11] Li Tao, Zhong Shisheng. Workflow model with colored timed Petri net and its performance analysis[J]. Journal of Computer-Aided Design \& Computer Graphics, 2006,18(6) : 821-831 (in Chinese)

[12] AALST W, HOFSTEDE A. YAWL : yet another workflow language[R]. Brisbane,Queensland,Australia : Queensland University of Technology, 2003

[13] W M P van der Aalst. The application of Petri nets to workflow management[J].Journal of Circuits, Systems and Computers , 1998, $8(1): 21-66$

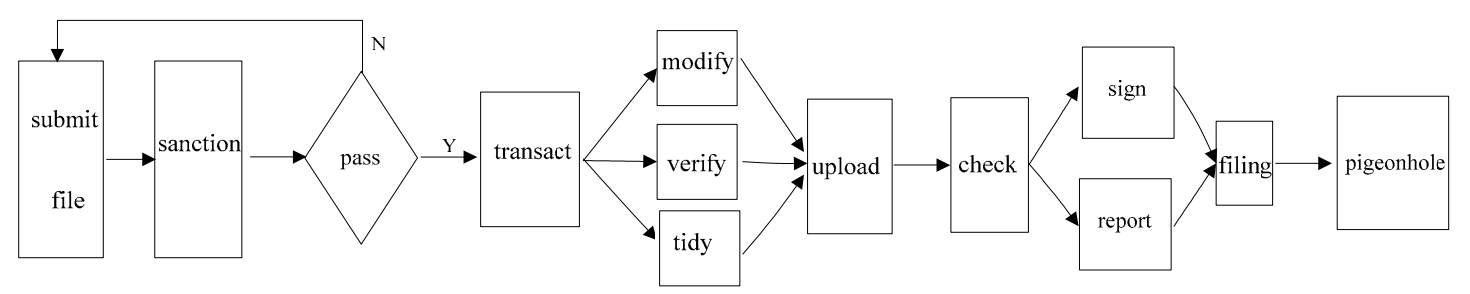

Figure 5. workflow model of sanction and management

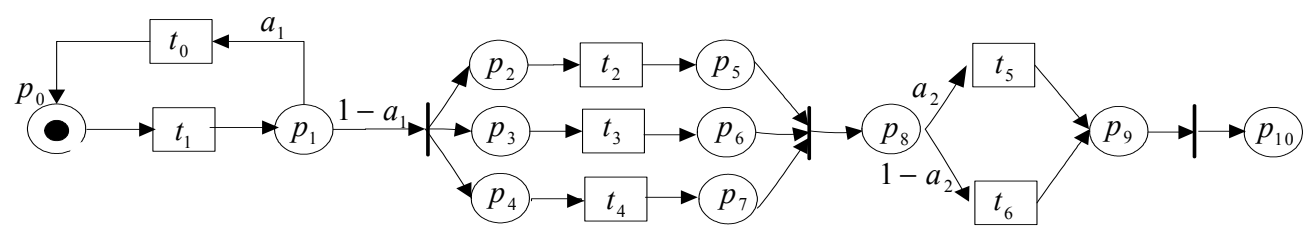

Figure 6. Workflow model basing on SPN

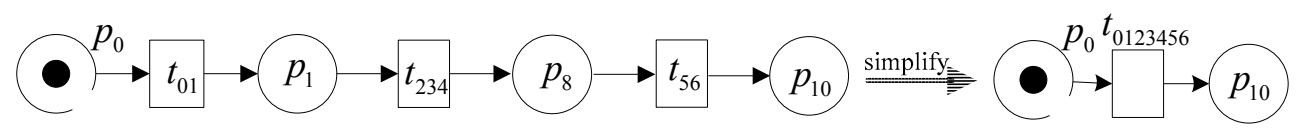

Figure 7. Simplification workflow model 